(Arts WFM et al. Follow-up of 146 children with epilepsy after withdrawal of antiepileptic therapy. Epilepsia May/June 1988; 29:244-250).

COMMENT. A relapse rate of 258 in this study is similar to that reported previously, and the relatively short treatment period did not appear to increase the risk of recurrence compared to studies requiring a minimum 4 year seizure-free period before discontinuing medicines. However, the absence of EEG epileptiform discharges at the time of drug withdrawal did not improve the outcome by reducing relapse rate compared to studies in which the EEG was not used in decision to withdraw. The results of this investigation emphasize the dangers of anti-epileptic drug withdrawal in mentally retarded female patients with neurological deficits.

\title{
READING EPILEPSY
}

Precipitating stimuli, including eye-movements, reading aloud versus reading silently, linguistic complexity, and concentration, were investigated in a 24 year old young woman with reading epilepsy seen in the Division of Neurology and Clinical Neurophysiology Laboratory, University of Ottawa General Hospital, and Depts of Psychology and Computer Engineering, Carleton University, Ottawa, Ontario, Canada. A viral illness at 19 years of age was followed by episodic jerking of the jaw while reading silently and a single generalized tonic-clonic seizure. After 2 years freedom from symptoms, the jaw jerking returned while reading, and was aggravated by fatigue or stress. Reading material was presented on a microcomputer video display monitor while the patient underwent EEG radiotelemetry video monitoring. Seizures were most readily elicited by reading aloud material of medium or high linguistic complexity. (Christie $S$ et al. Primary reading epilepsy: Investigation of critical-provoking stimuli. Epilepsia May/June 1988; 29:288-293).

COMMENT. Bickford et al, in their original description of reading epilepsy, observed precipitation of seizures by difficult reading matter (Trans Am Neurol Assoc 1956;81:100), and other authors have stressed the cortical or "lexical" aspects of the reading process in seizure provocation. The results of the present study suggest that a combination of factors involved in reading, including saccadic eye movements, articulation, and difficulty of linguistic content, contribute to epileptogenesis. Seizures were more readily provoked when greater demand was placed on each of the subtasks collectively and no factor acted solely as the critical stimulus.

In an article in the same issue of Epilepsia from the National Centre for Children with Epilepsy, Park Hospital for Children, Headington, Oxford, England, Verduyn et al surveyed mothers' impressions of seizure precipitants in children with epilepsy. Reading was not included in the list of precipitants, but stimuli that are somatic, psychological, and sensory were frequently followed by seizures. Mothers reported a relaxed state, emotional state, anxiety, exercise, and drowsiness as precipitants in 60-75\% of the sample of 446 children. Sensoryevoked epilepsy in $2.5 \%$ was provoked by loud noise, startle, flashing lights, bright light, diet, touch, television, and pattern. (Epilepsia 1988; 29:251). 